\title{
Photography Expression of Architecture of Qiang Nationality from the Perspective of Mobile Phone
}

\author{
Junhua Zeng ${ }^{1}$, Li Zhou ${ }^{2}$, Xin Tan ${ }^{1}$ \\ ${ }^{1}$ Art College, Southwest Minzu University, Chengdu, 610041, China \\ ${ }^{2}$ Architecture and Urban Planning College, Southwest Minzu University, Chengdu, 610041, China
}

Keywords: Photography perspective, Mobile phone photography, Architecture of Qiang nationality

\begin{abstract}
Nowadays, with the development of the interment, the mobile phone photography is taking up the mainstream culture of photography day by day. Its timeliness, convenience and transmission are unparalleled by other professional photography tools. How to improve the aesthetic and concept expression of the mobile photography art of the Qiang people's architecture is an urgent subject to be studied. This paper discusses the ideas, techniques and methods based on the mobile phone architecture photography, which makes the thinking of mobile architecture photography transform into the cornerstone of the practical communication. It analyzes the feasibility of mobile phone architecture photography, explores the new thinking of mobile phone architecture photography to make the architecture culture of Qiang nationality be better preserved, inherited and developed in the era of internet + mass media.
\end{abstract}

The Qiang architecture is an exotic flower in the history of China's architecture. Its external modeling is unique, with beautiful lines, reasonable space and perfect function. Qiang architecture is the cultural expression of Qiang's cultural form and connotation. Nowadays, with the development and dissemination of Qiang traditional culture and the development of Internet, people have explored the new media Qiang photography in the perspective of mobile photography.

\section{Photography Function Breakthroughs of Qiang people's Architecture from the Perspective of Mobile Phone}

\subsection{Application Function Breakthroughs of Mobile Phone Architecture Photography}

The product of new era of mobile phones has changed people's lives, cultural and artistic creation, communication and dissemination, especially brought new ways of innovation and expression for mobile phone photograph. The current mobile phone camera technology rose rapidly and various design and editing software technology combine to provide more convenient, high quality, ideal photographic image editing tool for our architectural photography creation, through the mobile phone rapid dissemination and promotion of image culture, has the function of limitation of timely and efficient. Secondly, combined with the photographer through the mobile phone lens saw Qiang architectural aspects and their culture connotation, emotional thinking, creative ideas, methods, the use of mobile phone image editing software, fast image formation in the first moment can be carried out and table design and image, and the recreation of image choice, achieve personalized photographer photography expression reproduction function. The other mobile phone photography using a wide area of our country, almost everybody has a mobile phone, when everyone is a photographer, is the era of big data cultural tourism participants, we can be more widely involved in recording history and culture, and Qiang people's building bit by bit, through the mobile phone network, WeChat, micro-blog, QQ and other publishing the cultural characteristics of Qiang nationality architecture and reproduced, further improve the radiation image feature phone Qiang architectural photography. In the current era of Internet big data and tourism culture development, with the help of mobile phone photography, the application function of the mobile phone digital 
media and the personalized expression of photographers will further expand the dissemination of Qiang Nationality's architectural culture and enhance the charm of Qiang national architectural culture.

In the past, the architectural photography in Qiang area was seen only in various promotional brochures and posters. It was only a small number of people involved in the dissemination of Qiang's architectural culture and art, and could not fully and widely reflect its cultural value. For the connotation of architectural designer and architecture, the artistic value cannot be comprehensively and accurately expressed, especially the detailed depiction, form, space, material, cultural details, emotion, artistic appeal and so on, which is narrow and deep, and cannot arouse the resonance of the masses. The breakthrough of mobile phone photography in the practical application of the Qiang nationality is first facing the cognition and aesthetics of the masses. People can share the perceived Qiang's architectural images and cultural customs on the Internet platform for the first time to spread, share and communicate with the Internet platform. At the same time, professionals also need to collect information about the Qiang building data quickly and effectively through the mobile media. In the future, with the development of the technology of mobile phone photography, more and more photographers will take up the cell phone to create a wider window for the artistic creation of Qiang's architectural photography.

\subsection{Mobile Phone Architecture Photography Breaks through the Transmission Pattern of Traditional Photography of Qiang People's Architecture}

Mobile photography blurred the boundaries of the dissemination and promotion of traditional architectural photography. In the dissemination of the Qiang nationality architecture image culture, the Qiang nationality architectural culture in the development and dissemination of the current planning of tourism cultural industry today, aging, fast and comprehensive communication and promotion is a necessary condition to seize the cultural characteristics of the Qiang nationality architecture propaganda and expand its influence opportunities, spread function and communication pattern and coverage wide hand camera in the Internet era, further reflects its fast, aging and influence, it has changed the way of transmission and the traditional pattern, make people more convenient to understand information and transmit information to a mobile phone as the publicity and promotion of national culture photography undoubtedly will develop in the future as the preferred. Using mobile phone photography can be ready to take the Qiang nationality architecture wonderful moment, whether it is the construction of local architectural style building, light perspective, symmetry, color, creativity can be a fast mapping, and pictures to micro-blog, WeChat and other social media platforms, for users and the media forwarding, thus faster and wider, further promote Qiang cultural construction, the communication method to accelerate the propagation effects of Qiang nationality architectural photography, change people watch and inherently transfer photos, expand the Qiang architectural culture propaganda and influence.

\section{Photography Expression of Architecture of Qiang Nationality from the Perspective of Mobile Phone Photography}

The Qiang's architectural photography of a new generation of positive expression image transformation and dissemination way, we need to express the kind of "freedom" in the national architectural photography creation process of the non-imprisonment new photography technology and the concept of photography, but the mobile phone photography as popular photography, forming techniques and conceptual photography is relatively lagging behind, how to enhance the expression of Qiang nationality architectural photography art aesthetic concept of innovation, mobile phone and media on the Internet, timeliness of communication at the same time make shooting skills more innovative, vision is more outstanding, comprehensive and accurate presentation of Qiang nationality architectural culture connotation and artistic features. 


\subsection{Photography Expression Innovation of Architecture of Qiang Nationality from the Perspective of Mobile Phone Photography}

In the light of the shadow, the exaggerated visual impact of the characteristics of the Qiang people's architecture. The Qiang architecture is tranquil, upright, persistent, magnificent and mysterious. The Qiang culture believes in all things. In the mysterious religious and cultural implication, all buildings are vague and clear, and the spirit and humanity are presented here. How to express its old and mysterious oriental Fort meaning and connotation? The creation of the use of mobile phone camera brings exaggeration and distortion performance of building tension, highlighting its architectural style characteristics, to contrast with the light and shadow color screen, the contrast of the visual impact on the screen to highlight the details of its architecture, reflect the pursuit of large capacity, creating space in great depth in depth. Strengthen the Qiang Minority Castle majestic and mysterious distant body feeling. During the filming of Qiang castle we often use carved side light and backlighting to show the architecture. The best side light is leaning at a right or left 45-degree angle above the Qiang's castle. In this light, the bright part of the Qiang's castle occupies $60 \%-70 \%$, and the shadow accounts for $30 \%-40 \%$. Currently the proportion of light and shade harmonization shows the momentum and beauty of the castle. The backlight can clearly outline contour of the Qiang Minority, is to strengthen the visual elements of the Qiang Minority form important, and all around as a whole show part of their beauty in the picture, a very important factor is the balance of composition, so that the composition proportion, to contrast the actual architectural conception and atmosphere, to light as the shadow highlight the theme, exaggerated to show the Qiang nationality architecture unique visual beauty, aesthetic image reflected the Qiang nationality architecture a simple atmosphere, is the current mobile phone and mobile phone digital photography communication era mainstream photography art form, not only can perfect shot expression and easy to spread and use of digital image in the internet era.

Blank daydream lets Qiang nationality architecture more ancient. Modelling and material characteristics of the Qiang architecture for orderly density and visual perception, and residential decoration level well-proportioned stone cutting layout closely to form a strong picture density rhythm. In the past, we often love photography, full picture composition, if not complete panorama of architectural expression, but it is full, full screen without blocking, give a person with imagination, appropriate blank reverie, extending to the picture of a stable and broad perception, but also the entire picture looks clean and tidy and remarkable quality. Mobile phone photography in the space of the entire blank screen can be controlled between two third and one third, is commonly used in the main composition proportion of blank, so you can focus on the performance of local characteristics and the Qiang nationality architecture, while the rest of the body empty blank form China context of traditional culture like expression, through the already formed like can unroll and reverie unlimited. Mr. Wu Guanzhong's works of the south of the Yangtze River is the simple modeling, many whitewash and limitless have a long and long taste. Photography and painting are the same. The context of "leaving white" and "meaning" can show the quintessence of Qiang's ancient Qiang culture. In the Qiang Nationality's architecture, its physical quantity or building material decoration shows a close and full sense to people. Deliberate white space can not only express the characteristics, but also keep the imagination space, so that the Qiang culture will go far back.

Lines and local details highlight the art and artistic charm of the Qiang people's architecture. In mobile photography, line is an important element in the expression of photography. In morphology, the lines are straight and curved. They are roughly divided into vertical lines, horizontal lines, oblique lines, crossover lines, curves, and circular lines. These lines have their own beauty and characteristics, expressing specific artistic conception and artistic effects. In addition to the grand building of Qiang overall, there is a wealth of line and detail, regardless of appearance or internal structure, lines are unique to each other, the vertical and horizontal, inclined, cross, have arranged in a crisscross pattern the depth of feeling, give a person a positive, tall and magnificent architectural impression, reflect different artistic conception and art effect. In the Qiang building doors and windows wooden structure, white stone expression, totem pattern decoration and so on rich and colorful lines, or 
vertical, horizontal and inclined, cross, or curve, circular, many wonderful linear expression and exquisite details to Speechless incomparable gorgeous, so the mobile phone camera these wonderful lines, or local details can give the audience a great visual impact. Now, in the mobile phone in our Qiang architectural photography shooting mode and route of transmission from the traditional form of distinction, through this line and local fine shooting can be found exquisite craft and other more Qiang architectural details of visual art, the formation of resonance, dissemination and promotion in mass media art, thus the correct interpretation of Qiang nationality the building of ancient traditional craftsmanship and artistic charm.

\subsection{Innovation of Self-Photography View of Qiang People's Architecture Photography from the Perspective of Mobile Phone Photography}

In the architectural photography of the Qiang people, we also need a picture of the image. Therefore, we need to show the photographer's creative ideas, thoughts, expressive force and material carrier is not the same, because at the same time the photographer formed in their own ideas determines the cultural connotation and characteristics of the painting style of expression, he can choose to cut the viewfinder, the composition and the material medium of expression, through the relationship, picture the actual strength and contrast the Qiang nationality architecture of three-dimensional shape and texture and visual feeling, create a distant and mysterious ancient Qiang architecture vivid artistic expression. The proportion of local perspective to exaggerate the Qiang nationality architecture to reveal their individual characteristics to the performance of construction, strengthen the artistic appeal to obtain the typical visual aesthetic expression of Qiang nationality architecture, view Qiang architectural photography embody self-photography from the perspective of innovation and mobile phone. Thus for building mobile phone photographers internal cultivation, exercise form and update and art concept, for photography Qiang architecture has essential significance, we know the concept of a person's thoughts and ideas of decision, expression of media materials innovation use but can give a person find everything fresh and new visual experience, and can only form his creation concept the original concept of boundaries and overcome the renewal of solidification, so as to change the backward ideas and technology, not in the rules, use of new ideas and new methods of science and technology innovation of mobile phone photography and Internet era in the current to explore the Qiang nationality architecture photography profound national architectural art.

\section{Conclusions}

The architectural photography of the Qiang people of the mobile phone has made up the vacancy of the traditional Qiang people's photography, which provides a wider field for the spread and promotion of the Internet era. In today's digital era Qiang architecture photography art with modern mobile phone photography technology, through the exploration of Qiang nationality architectural theme photography deeply Qiang area theme selection, discover the classic characteristics of the Qiang nationality architecture, combined with the artist's idea, theme and artistic expression for a series of Qiang architectural masterpiece. The Qiang people's architectural photography art has seized the historical development and transformation period, and is brave in exploring, learning and broadening the way of thinking. Through mobile phone photography, it is more efficient and efficient in cultural dissemination, publicity and promotion. Combining photography techniques and aesthetics principle enhance the traditional mobile phone photography image of Qiang architectural aesthetics and cultural implication, the concept of innovation, we let the mobile phone to be able to participate in the construction of real Qiang photography, witness, upload, share and reflect the value of its own evidence, plays a certain role in the inheritance and spread of Qiang architectural culture. With the rise of tourism, the opportunity to witness the shooting of Qiang people's architecture is more frequent, strong interaction and increasing influence. A culture needs to be informed, carried out, understood and spread to better promote its development. In this era of Internet, its influence and communication power cannot be ignored. In this time, the Qiang architectural culture should further 
promote and promote itself, and let the Qiang architectural culture go out of the Qiang mountain and go to the world.

\section{Acknowledgements}

This paper is the periodical results of Central University Project of Southwest Minzu University named "Architecture of Qiang Nationality from the Perspective of Photography" (Grant No. 2016szyqn57).

\section{References}

[1] Wang Wei. The Application of Mobile Phone Addiction to Teaching Methods - A Case Study of Photography Practice Teaching [J]. Education Teaching Forum, 2017(37): 5-7.

[2] Xiang Weidong, Zhang Chu. Camera Phone and Mobile Phone Photography in the Media Convergence [J]. Journal of Chongqing Normal University Edition of Social Sciences, 2012(5): 60-64.

[3] Chen Chen. Technology Creates the Aesthetic Connotation of Mobile Phone Photography [J]. The Guide of Science \& Education, 2017(17): 153-154.

[4] Gao Jia. Mobile Photography in Mobile Internet Era - I understand about phone photography [J]. Openings, 2015(5): 18-21. 